Lambo, T. A. (1956). Brit. med. J., 2, 1388.

- (1960). Ibid., 2, 1696

Laubscher, B. J. F. (1951). Sex, Custom, and Psychopathology. Routledge and Kegan Paul, London.

McGeorge, J. (1942). Med. J. Aust., 1, 67.

Mahi, Tigani El (1959). Africa: Social Change and Mental Health. W.H.O.

$\emptyset$ degard, Ø. (1932). Acta physiol. scand., Suppl. 4, p. 11.

Read, C. S. (1936). Brit. med. J., 1, 631.

Sainsbury, P. (1955). Suicide in London. Chapman, London.

Stengel, E. (1950). Recent Progr. Psychiat., 2, 691.

and Cook, N. G. (1954). J. forens. Med., 1, No. 5.

Tooth, G. (1950). Studies in Mental Illness in the Gold Coast. Colonial Research Publ. No. 6. H.M.S.O., London.

Wicksell, S. (1934). Svenska Forenigrens for Psykisk Halsovard Smaskrifter, No. 7. Quoted by Dahlgren (1945).

Yap, P. M. (1958). J. ment. Sci., 104, 266.

\section{AN ABNORMAL CHROMOSOME IN CHRONIC LYMPHOCYTIC LEUKAEMIA*}

\author{
BY
}

\section{F. W. GUNZ, M.D., Ph.D., M.R.C.P.}

\section{P. H. FITZGERALD, M.Sc.}

AND

ANGELA ADAMS, B.Sc.

From the Cytogenetics Unit, Christchurch Hospital, Christchurch, New Zealand

The somatic chromosomes of patients with chronic lymphocytic leukaemia have hitherto not been extensively studied, and there are no published accounts of any consistent abnormalities in this disease. This contrasts with the chronic granulocytic variety in which a characteristic defect of one of the small acrocentrics,

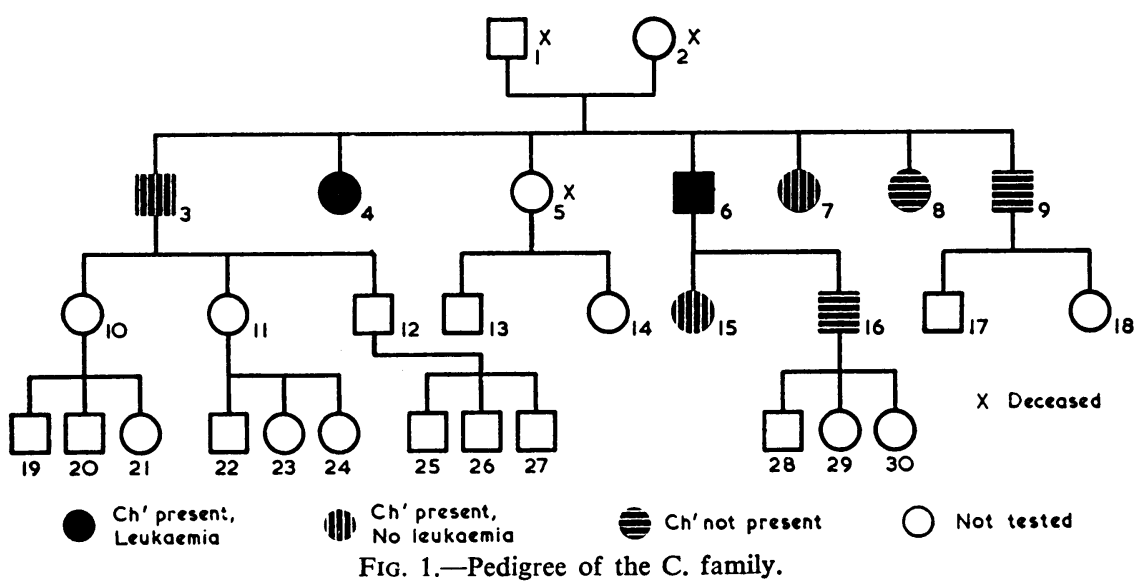

the so-called $\mathrm{Ph}^{1}$, has been found in most if not all cases (Baikie et al., 1960; Nowell and Hungerford, 1960, 1961 ; Tough et al., 1961). We now report the discovery of a new chromosome abnormality differing from the $\mathbf{P h}^{\mathbf{1}}$ but also affecting a small acrocentric, probably No. 21 in the Denver classification (Lancet, 1960). This occurred in a family in which two of the members have chronic lymphocytic leukaemia. In addition, we have found a similar abnormality in another individual with chronic lymphocytic leukaemia whose brother had died of the same disease. In accordance with the convention adopted at the Denver meeting, we propose the term $\mathrm{Ch}^{\mathbf{1}}$ for the abnormal chromosome.

*This investigation was carried out with the support of the Canterbury Division of the British Empire Cancer Campaign Society.
In this investigation all the chromosomes examined were derived from blood cultures made by the method of Moorhead et al. (1960). Direct bone-marrow preparations were also studied, but because of the paucity of mitoses in marrows heavily infiltrated with lymphocytes these were unsatisfactory.

\section{The C. Family}

The pedigree of this family is shown in Fig. 1. No. 1, the father, died in 1926 from "sarcoma of the mediastinum," but no records survive of his illness, other than the death certificate, and a necropsy was not performed. The mother, No. 2, died in 1943 from a cerebrovascular accident. The propositus, No. 4, aged 67, was found to have chronic lymphocytic leukaemia in February, 1962. Cultures of her blood were made before the institution of her treatment. Her brother, No. 6, aged 64, had been known to have chronic lymphocytic leukaemia since 1954 and had been treated first with splenic $x$-irradiation and subsequently with chlorambucil ("leukeran"). His blood was cultured in 1962. One sister, No. 5, died in Australia, aged 50, from carcinoma of the uterus; she was said to have had pernicious anaemia. The other siblings are alive and well, but one, No. 7, now aged 60 , is mentally defective, and another, No. 3 , has recently had severe trigeminal herpes zoster. All members of the next two generations are healthy.

\section{The Chromosome Abnormality}

Cultures were prepared from the blood of Nos. 3, 4, $6,7,8,9,15$, and 16 . The modal chromosome number in all cases was 46 . The abnormal chromosome, $\mathrm{Ch}^{1}$, was present in the two patients with leukaemia, and in Nos. 3, 7, and 15 (Fig. 1). In the positive cases it was found in nearly all cells which could be clearly analysed ; it was doubtfully present in a few cells, but definitely absent in none. We conclude that its true incidence was $100 \%$.

The chromosome is shown in Figs. 2 and 3. It was one of the four small acrocentrics, and the abnormality consisted in the complete or almost complete loss of the short arm, with apparent preservation of the centromere and long arm. The effect was to give the chromosome the appearance of a horseshoe or, in slightly contracted specimens, of a dumb-bell. In still more contracted figures the two arms became oval knobs lying either side by side or sometimes at 180 degrees to each other.

The $\mathrm{Ch}^{1}$ chromosome was not found in nine other patients with chronic lymphocytic leukaemia whose blood was cultured. This abnormality, or a closely related one, was, however, present in the cells cultured from the blood of another patient with chronic lymphocytic leukaemia whose brother had died in January, 1962 , from the same disease. The defective acrocentric chromosomes in this case showed a small remnant of the short arm, whereas no such remnant could be seen in those of the members of the $C$. family. 


\section{Discussion}

The present report is the first in which an inherited chromosome defect has been found to be associated with the occurrence of chronic leukaemia. Because of the great rarity of familial leukaemia (Dameshek and Gunz, 1958) and the apparent absence of the new chromosome abnormality in other conditions, it appears reasonable at this stage to regard their association as more than merely fortuitous. In our cases the $\mathrm{Ch}^{1}$ chromosome was present in $100 \%$ of mitotic figures which could be analysed (a total of 237 in five individuals). It was inherited and must therefore have also been present in somatic cells other than leucocytes. In this it differed from the $\mathrm{Ph}^{1}$ defect in chronic granulocytic leukaemia which occurs only in cells of the granulocyte series of leucocytes (Tough et al., 1961) and, so far as is known, is not inherited. It is of interest that both abnormalities affect one of the small acrocentric chromosomes, although not the same part. When it is further remembered that No. 21 is trisomic in most cases of mongolism, a condition associated with an incidence of leukaemia nearly twenty times normal (Stewart et al., 1958), the case becomes strengthened for a connexion

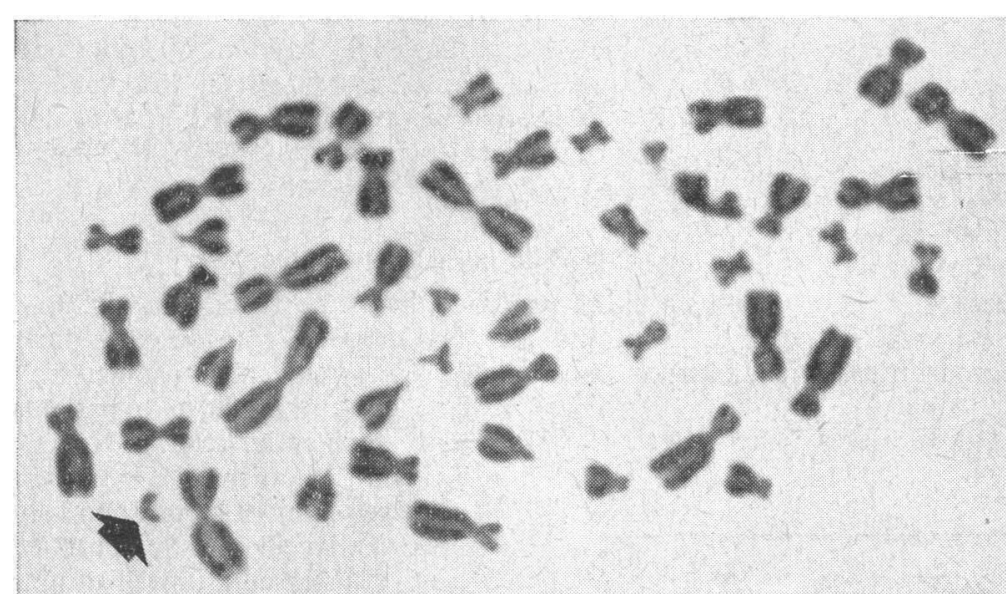

Fic. 2.- Chromosomes of a cell from a three-day culture of the blood of patient No. 7 (see Fig. 1). The $\mathrm{Ch}^{1}$ chromosome is indicated by an arrow.

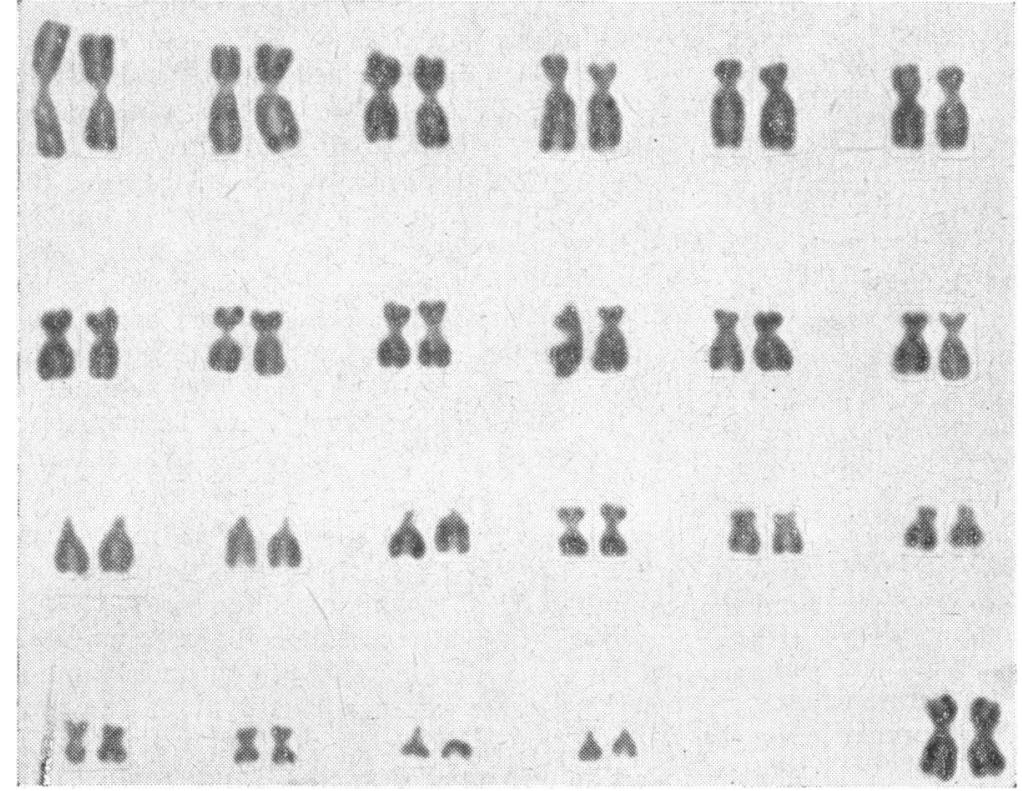

Fig. 3.-Karyotype of the cell shown in Fig. 2. The $\mathrm{Ch}^{1}$ chromosome is shown in the No. 21 position. between the small acrocentrics and the regulation of leucopoiesis, as first suggested by Tough et al. (1961).

The mechanism leading to the loss of a substantial part of the chromosome is not clear; it could be a deletion or a translocation, and we have no evidence enabling us to make the distinction. We have found the defect so far only in cases of " familial " leukaemia, a rare but well-recognized form. As more cases are examined it is possible that it may be seen also in patients who have no familial background of their disease. The $\mathrm{Ch}^{1}$ is a very striking abnormality ; minor, less obvious defects of the same chromosome region may well be discovered in the course of further work now in progress; if visible at all, these would still be of great importance in terms of loss of genic material.

The $\mathrm{Ch}^{1}$ was present in several individuals who, so far as we could determine, did not have leukaemia. They were clinically well and their blood counts were normal. One of them (No. 15) was only 33 years old, but the others were well within the age-group in which chronic lymphocytic leukaemia commonly occurs. In these the loss of translocation of a substantial part of a chromosome has so far led to no ascertainable clinical defects ; the affected chromosome region must thus be genetically rather " silent." Any of these patients may develop leukaemia in the future; however, if, as seems likely, the chromosome abnormality is involved in the production of the disease, it is evidently not by itself sufficient to cause it; rather it appears to predispose to the onset of leukaemia, much as does the trisomy of No. 21 in mongolism (though in the latter case in a much smaller proportion of those carrying it). For the production of overt leukaemia one or more factors besides the presence of the $\mathrm{Ch}^{1}$ are necessary. These could include age, hormones, external carcinogens, viruses, and others.

Chronic lymphocytic leukaemia differs from other forms of the disease in several respects. It has a striking racial distribution, being virtually non-existent in some nen-Caucasian populations such as the Japanese. In patients suffering from it there is a strong association with personal cancer, past or concurrent, and a possible excess of a family history of cancer. These and other findings have been thought suggestive of a genetic background in at least some cases of the disease (Gunz, 1961). This hypothesis is strengthened by the finding, so far in one family and possibly in a second, of an inherited chromosome abnormality associated with chronic lymphocytic leukaemia. Since the abnormality must be present in epithelial tissues as well as in those derived from the mesoderm, the high incidence of cancer in such patients might also be 2 consequence of a genetically determined predisposition. 


\section{Summary and Conclusion}

Two siblings with chronic lymphocytic leukaemia, as well as several other members of the same family without clinical leukaemia, were found to have a striking chromosomal abnormality in their leucocytes. The abnormality was not seen in nine other patients with chronic lymphocytic leukaemia, but the same or a closely related one was present in a further patient who had lost a brother from the same disease. The abnormality, for which the term $\mathrm{Ch}^{1}$ is suggested, appears to predispose individuals carrying it to the onset of chronic lymphocytic leukaemia.

AdDENDUM.-Further investigation has shown the $\mathrm{Ch}^{1}$ chromosome to be present in No. 12 and absent in No. 10 (Fig. 1). Miss Jean Ferguson, of the Commonwealth Serum Laboratories, Melbourne, has kindly cultured and examined skin cells of Nos. 4 and 6 . In both cases the $\mathrm{Ch}^{1}$ chromosome was present in all cells which were suitable for examination.

\section{REFERENCES}

Baikie, A. G., Court-Brown, W. M., Buckton, K. E., Harnden, D. G., Jacobs, P. A., and Tough, I. M. (1960). Nature (Lond.), 188, 1165.

Dameshek, W., and Gunz, F. (1958). Leukemia. Grune and Stratton, New York.

Gunz, F. W. (1961). Brit. med. J., 1, 326.

Lancet, 1960, 1, 1063.

Moorhead, P. S., Nowell, P. C., Mellman, W. J., Battips, D. M., and Hungerford, D. A. (1960). Exp. Cell. Res., 20, 613.

Nowell, P. C., and Hungerford, D. A. (1960). Science, 132, 1497. - (1961). J. nat. Cancer Inst., 27, 1013.

Stewart, A., Webb, J., and Hewitt, D. (1958). Brit. med. J., 1, 1495.

Tough, I. M., Court-Brown, W. M., Baikie, A. G., Buckton, K. E., Harnden, D. G., Jacobs, P. A., King, M. J., and McBride, J. A. (1961). Lancet, 1, 411.

\section{Preliminary Communications}

\section{Cardiac Complications after Vaccination for Smallpox}

Reports of cardiac complications following smallpox vaccination are rare (Cangemi, 1958). However, during the recent outbreak of smallpox a death was reported from acute focal myocarditis 11 days after vaccination (Mant, 1962), and about this time three patients who had recently been vaccinated presented in one hospital with cardiac involvement. Although it cannot be proved conclusively that these illnesses were caused by vaccination the presumptive evidence is strong enough to suggest that the risk of cardiac complications has been previously overlooked, and it seems desirable, now that widespread vaccination is again advocated, that these cases should be reported.

\section{CASE 1}

The patient, a man of 49 , was in good health prior to vaccination, his first. Ten days after this he developed rigor, headache, and malaise. These symptoms subsided after two days, but on the thirteenth day after vaccination he developed a constant dull aching pain behind his upper sternum with slight radiation into the left upper chest. This pain, which bore no relation to posture or exercise, persisted for three days but disappeared spontaneously on the sixteenth day, the day before he was admitted to hospital for investigation.
On examination he looked pale. There was no pyrexia. The pulse was 80 and the blood-pressure $105 / 60 \mathrm{~mm}$. $\mathrm{Hg}$. The heart was not enlarged and sounded normal. There were no murmurs or friction rubs. The electrocardiogram on admission showed S-T segment elevation in leads I, II, and V2-V4. The T wave was inverted in lead AVL and flattened in lead $I$. The sedimentation rate was raised to $35 \mathrm{~mm}$. (Westergren) in one hour. The serum glutamic oxaloacetic transaminase (S.G.O.T.) was normal and was reported as 26,25 , and 30 units on consecutive days after admission. Radiological examination of the heart and lungs was normal.

The patient's progress in hospital was asymptomatic. A week after admission the $S-T$ segment elevation in the precordial leads had disappeared and the inversion of the T wave in lead AVL was less pronounced. The bloodpressure had risen to $135 / 70 \mathrm{~mm}$. $\mathrm{Hg}$.

After discharge from hospital he continued well and his electrocardiogram returned to normal with upright $T$ waves in lead $I$ and flat $T$ waves in lead AVL.

CASE 2

A man of 57 was in good health prior to being revaccinated for the first time. On the seventh day his arm was painful and he had rigors and malaise. He subsequently improved until the thirteenth day after vaccination, when he found he was very breathless going upstairs. He had no chest pain or discomfort but vomited once before his admission to hospital on the fourteenth day.

On examination he had a pyrexia up to $102^{\circ} \mathrm{F} .\left(38.9^{\circ} \mathrm{C}\right.$. $)$ which persisted for four days. The pulse was irregular at about 120 owing to atrial fibrillation. The blood-pressure was $100 / 80$ $\mathrm{mm}$. Hg. Pericardial friction rubs were audible to the left of the lower end of the sternum and persisted for $\mathbf{4 8}$ hours. There was a marked local reaction at the site of vaccination on the left arm, with enlarged axillary lymph nodes. There were no abnormal physical signs in the chest. The electrocardiogram on admission showed atrial fibrillation. There was no evidence of cardiac infarction or of the pericarditis. The sedimentation rate was raised to $35 \mathrm{~mm}$. (Westergren) in one hour. The haemoglobin was only $76 \%$, but the blood was otherwise normal. The S.G.O.T. was normal on three successive days. Radiological investigation of the chest showed slight loss of translucency in the right mid-zone on admission, but this had disappeared 14 days later. The cardiothoracic ratio on the first film was $18 / 31.5 \mathrm{~cm}$. and $17 / 31 \mathrm{~cm}$. on discharge in sinus rhythm.

Initially this patient's arrhythmia was regarded as a manifestation of "silent cardiac infarction" and he was treated with heparin and phenindione. However, after a week this was stopped when the diagnosis was revised and the arrhythmia was regarded as a complication of his vaccination. He was restored to sinus rhythm with quinidine and discharged from hospital. He returned to work three weeks after the onset of his breathlessness and has remained well since. Several normal electrocardiograms have been recorded.

\section{CASE 3}

A woman of 37 was subjected to a right oophorectomy and a left ovarian cystectomy on the second day after a primary vaccination. On the third post-operative day, five days after vaccination, she had a sudden attack of breathlessness lasting for about 15 minutes, associated with severe pain in her right lower chest.

On examination at this time the pulse was 120 and the blood-pressure $110 / 75 \mathrm{~mm}$. Hg. There were no abnormal signs in the heart or chest and no evidence of venous thrombosis in the legs. $X$-ray examination of the chest revealed nothing abnormal. An electrocardiogram was grossly abnormal and showed inverted $T$ waves in leads $I$ and II, lead AVF, and precordial leads V1-V6. It was not thought possible that such widespread electrocardiographic changes could have been caused by a pulmonary embolus, 